\title{
Modeling and Simulation of Organic Field Effect Transistor (OFET) Using Artificial Neural Networks
}

\author{
Imad Benacer and Zohir Dibi \\ Electronics department, University of Batna, Algeria \\ benacerimad@gmail.coml
}

\begin{abstract}
This paper presents the modeling and simulation of a low voltage pentacene organic field effect transistor (OFET), which is based on experimental data using an integrated finite element and artificial neural networks (ANN) approach. We present a model of organic field effect transistor based on neural network, this approach allows an easy way to model devices without acquiring a deep knowledge the device physics. The finite element type simulation is realized using 2-D Atlas simulator, both Atlas and obtained modeling results agree approximately with the published experimental results.
\end{abstract}

Keywords: Organic Field Effect Transistors (OFET), Organic Semiconductor (OSC), Channel Length, Poole-Frenkel mechanism, Artificial Neutral Networks (ANN)

\section{Introduction}

Research on organic field effect transistors (OFET) has been rapidly growing in recent years. Owing to lightweight, low cost, and low fabrication temperature, organic field effect transistors (OFETs) have a wide range of applications such as sensors, active-matrix backplanes in displays, and radio frequency identification tags (RFIDs) [1-2]. Recently pentacene-based thin-film transistors (TFTs) have seen significant improvements in device performance. Organic TFT performance is now comparable to hydrogenated amorphous silicon (a:Si:H) TFTs [3]. The performance of organic transistors depends largely on the quality of the insulator/organic interface, the gate insulator, the morphology of the organic thin film, and the charge injection process. Organic transistors are working in accumulation mode and most of the modulated charge of the conducting channel resides in the first monolayer next to the insulator-semiconductor interface [4]. This means that the interface properties between the semiconductor and the gate dielectric are of tremendous importance on the field effect mobility.

Roughness, density of surface traps, and dielectric constant are crucial parameters [5]. Among inorganic dielectrics, most OFETs utilize an insulator made of an oxide (mainly silicon oxide $\mathrm{SiO} 2$ ), this kind of OFET requires a relatively high voltage for operation, on the other hand the weak of dielectric constant $(\varepsilon=3.9)$ remains a serious limitation for low power since operating voltage largely above $50 \mathrm{~V}$ are necessary for sufficient charge injection in the channel. To allow organic TFTs to operate with low voltages and hence the power consumption, high-K materials such as $\mathrm{Al}_{2} \mathrm{O}_{3}[6]$ and $\mathrm{Ta}_{2} \mathrm{O}_{5}$ [7] have been employed as a gate dielectric to fabricate OFETs.

Device modeling for circuit simulation is usually done through compact models, which tray to model the physical phenomena inside the device that uses either physically based functions or empirical functions [8]. When fast modeling is required and we are not concerned about the physics inside the device, the easy modeling approach in dealing with 
nonlinear systems is to use non-linear methods such as artificial neural networks (ANN). A very common application of ANNs is in control systems where the system is non-linear and the exact relationship is difficult or impossible to determine analytically. Artificial neural networks are pliable mathematical structures that are capable of identifying complex nonlinear relationships among input and output data sets.

\section{Simulation}

\subsection{Finite element based Atlas simulation}

To make OFETs operating at lower voltages $(2-3 \mathrm{~V})$, with a typical dielectric permittivity ( $\varepsilon \sim 3-5$ ), the gate capacitance should be close to $0.5 \mu \mathrm{F} / \mathrm{cm}^{2}$. This means that the gate insulator should be less than $10 \mathrm{~nm}$ thick [9].

The structure of top contact device simulated is shown in Figure 1, these devices have channel length $\mathrm{L}$ of $10 \mu \mathrm{m}$ and a channel width $\mathrm{W}$ of $100 \mu \mathrm{m}$, with $30 \mathrm{~nm}$ thick film of pentacene and $30 \mathrm{~nm}$ thick gold source/drain contacts, where the gate electrode was 20 $\mathrm{nm}$ of Aluminum and dielectric gate thickness of $5.7 \mu \mathrm{m}$ was used for simulation as mentioned in [9]. In finite element based simulation, the Poole-Frenkel mobility is used for Pentacene layer and defines the dependency of mobility capability due to electric field, this model is expressed as

$$
\mu(E)=\mu_{0} \exp \left(-\frac{\Delta E_{a}-\beta \sqrt{E}}{K_{B} T}\right) .
$$

Where $\mu(E)$ is the field dependent mobility, $\mu_{0}$ is the zero field mobility, $\Delta \mathrm{E}_{\mathrm{a}}$ is The zero field thermal activation energy which is equal to $0.018 \mathrm{eV}, \mathrm{E}$ is the electric field, $\mathrm{K}_{\mathrm{B}}$ is the Boltzmann constant, $\mathrm{T}$ is the temperature and $\beta$ is The Poole-Frenkel factor which is equal to $7.75 \times 10^{-5} \mathrm{eV}(\mathrm{cm} / \mathrm{V})^{0.5}$ [9-12]. To perform numerical simulation, typical pentacene properties are shown in Table 1, Experimental value of interface trap state density of $3 \times 10^{12} \mathrm{~cm}^{2}[9]$ was used for the simulation.

Table 1. Pentacene properties for OFET device

\begin{tabular}{|c|c|c|}
\hline Material (layer) & Parameter & Value \\
\hline & Band gap & $2.2 \mathrm{eV}$ \\
& Density of conduction band & $2 \times 10^{21} \mathrm{~cm}^{-3}$ \\
Pentacene & Density of valence band & $2 \times 10^{21} \mathrm{~cm}^{-3}$ \\
& Permittivity & 4.0 \\
& Acceptor doping concentration & $7 \times 10^{17} \mathrm{~cm}^{-3}$ \\
& Affinity & $2.8 \mathrm{eV}$ \\
\hline Gate insulator & Dielectric constant & 4 \\
\hline
\end{tabular}

In Poole-Frenkel model, conduction occurs due to the field enhanced thermal excitation of trapped charge carriers. The drain current reduces in low field region, due to charge carriers being localized around the traps. Increasing the electric field will help these trapped charges to exit the trap. The release of these charge carriers will result in a large increase in the drain current of the device [13].

Figure 1 shows simulated structure for bottom gate top contact (BGTC) device and it can be easily understood from current flow lines, that charge injection/extraction is taking place 
from side/corner of the contacts and charge transport occurs in the first few layers adjacent to the dielectric. The output and transfer characteristics are examined for top contact OFET, and the simulated results are shown in Figure 2 and Figure 3 respectively. As indicated in Figure 2 drain current $\left(\mathrm{I}_{\mathrm{dmax}}\right)$ is $4.94 \mu \mathrm{A}$, at specified gate voltage and drain voltage of $-3.0 \mathrm{~V}$.

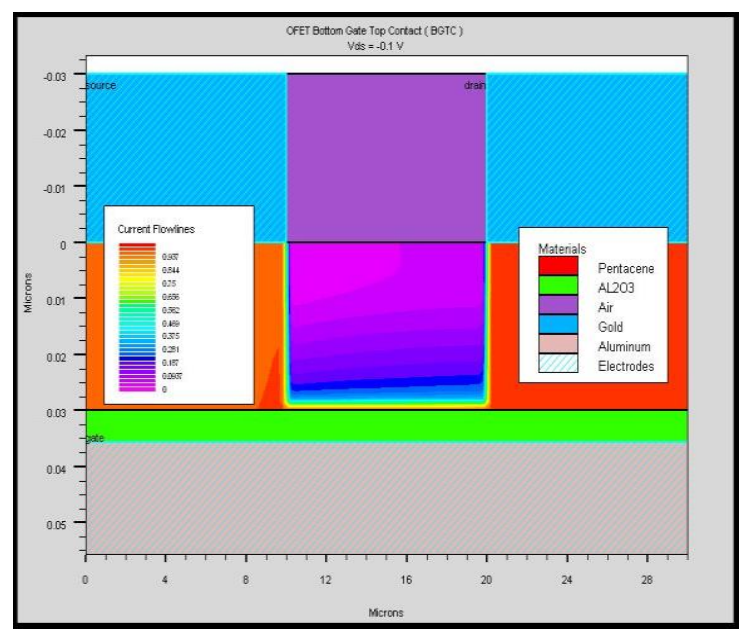

Figure 1. Schematic diagram for current flow path of top contact OFET structure

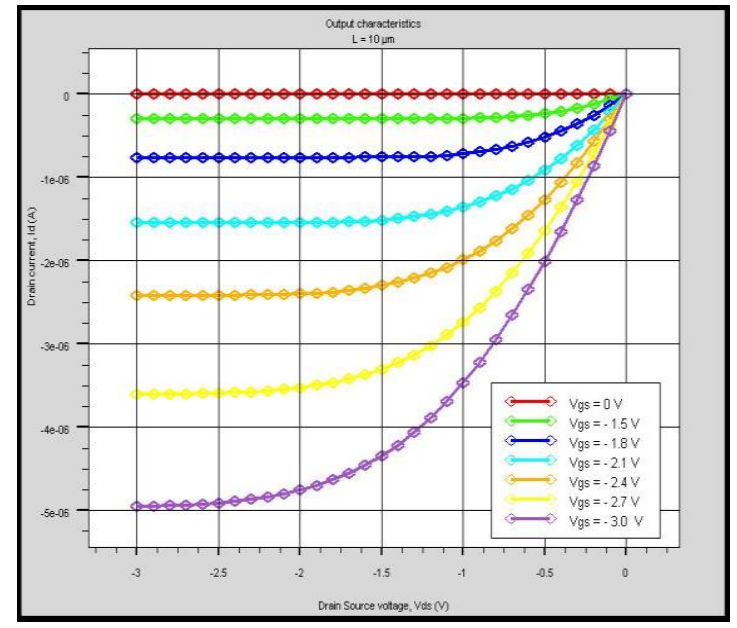

Figure 2. Output characteristic with channel lengths of $10 \mu \mathrm{m}$

We have simulated the transfer curve for different values of $\mathrm{L}$ ranging from 10 to $50 \mu \mathrm{m}$ (Figure 3). Simulation results show that the increase in drain current accompanying the decreasing of the channel length. Figure 4 shows potential profile at $1 \mathrm{~nm}$ above the semiconductor/dielectric interface along the device length, the potential was simulated at $\mathrm{V}_{\mathrm{GS}}=\mathrm{V}_{\mathrm{DS}}=-3 \mathrm{~V}$. 


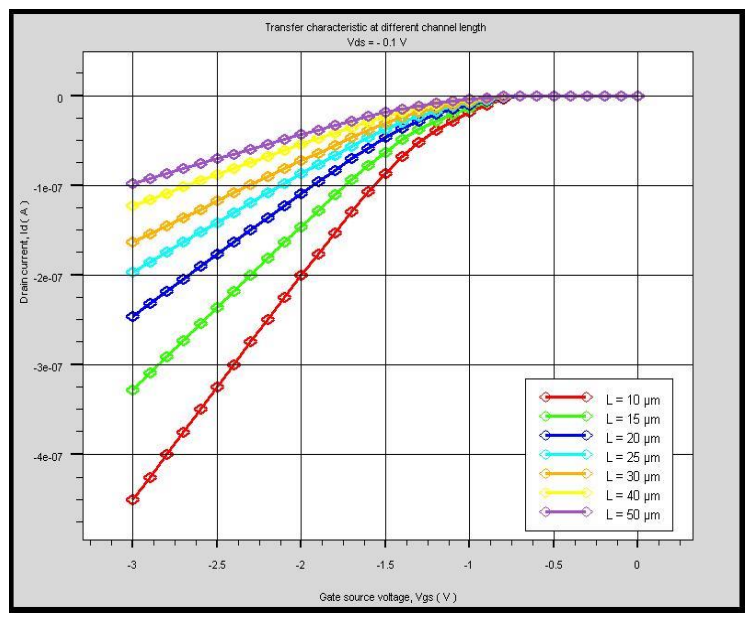

Figure 3. Transfer characteristics (ID-VGS) at different channel lengths

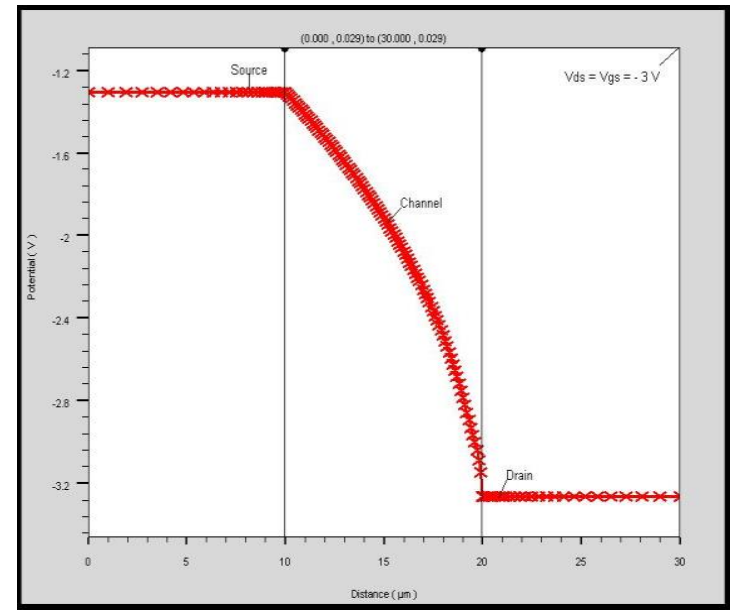

Figure 4. Potential profile in pentacene for $10 \mu \mathrm{m}$ channel length

\subsection{Artificial Neural Networks}

The artificial neural network (ANN) is a system of data processing based on the structure of a biological neural system. An ANN is a powerful data modeling tool that is able to capture and represent complex input/output relationships by some internal calculations. The most common neural network model is the multilayer perceptron (MLP) [14-15] shown in Figure 5. A typical neural network structure has two types of basic components, namely, the neurons and links between them. Every link has a corresponding weight parameter associated with it. Each neuron consists of a set of inputs, and an activation function. The network architecture is composed of an input layer $(\mathrm{X})$, an output layer $(\mathrm{Y})$ and one or more hidden layers $(\mathrm{H})$. MLP is a feed forward ANN model that maps sets of input data onto a set of appropriate output. A simplified overview of the proposed ANN model is shown in Figure 6.

Equation 2 represents the algorithm commonly used to compute the output of an artificial neuron. The output of each neuron $\mathrm{f}$, depends on the activation value, which is a weighted sum of the inputs 


$$
f(x)=k\left(\sum\left(x_{i} w_{i}\right)\right)
$$

Where $\mathrm{w}_{\mathrm{i}}$ is the weight related to the $\mathrm{x}_{\mathrm{i}}$ input and $\mathrm{f}$ is the activation function.

The MLP networks are trained with the standard back-propagation algorithm, which compares the predicted output with the desired output and the mean square error is calculated. If the mean square error is more than a prescribed threshold value, it is back propagated from output to input, and weights are further modified until the error or number of iterations is within a prescribed limit. The training of a network is a process where the set of adjusted parameters (weights and biases) is optimized in order to make the best prediction of the target variable on the basis of background variables [16].

The neural network model was created in MATLAB (2006a). The Network properties are as follows:

- Network inputs: $\mathrm{V}_{\mathrm{GS}}, \mathrm{V}_{\mathrm{DS}}, \mathrm{L}$ and $\mathrm{t}$.

- Network outputs: $\mathrm{I}_{\mathrm{D}}$.

- Network type: Feed-Forward Back-Propagation.

- Network transfer function: logsig

- Training function: TRAINRP.

In order to compare the results of the proposed ANN model in modeling the organic field effect transistor with experimental results, we have used a relative error (RE) measurement which is expressed in percentage defined as:

$$
\mathrm{RE} \%=\frac{\mathrm{I}_{\operatorname{Exp}}-\mathrm{I}_{\text {Pred }}}{\mathrm{I}_{\mathrm{Exp}}} \times 100
$$

where $\mathrm{I}_{\text {Pred }}$ is the predicted drain current based on ANN, $\mathrm{I}_{\text {Exp }}$ represents the target function (experimental measures), RE gives an indication of how good a measurement is relative to the size of the thing being measured. Also the mean relative error (MRE) is given by:

$$
M R E=\frac{1}{N} \sum_{i=1}^{N}|R E|_{i} .
$$

where $\mathrm{N}$ is the number of data (database size). 


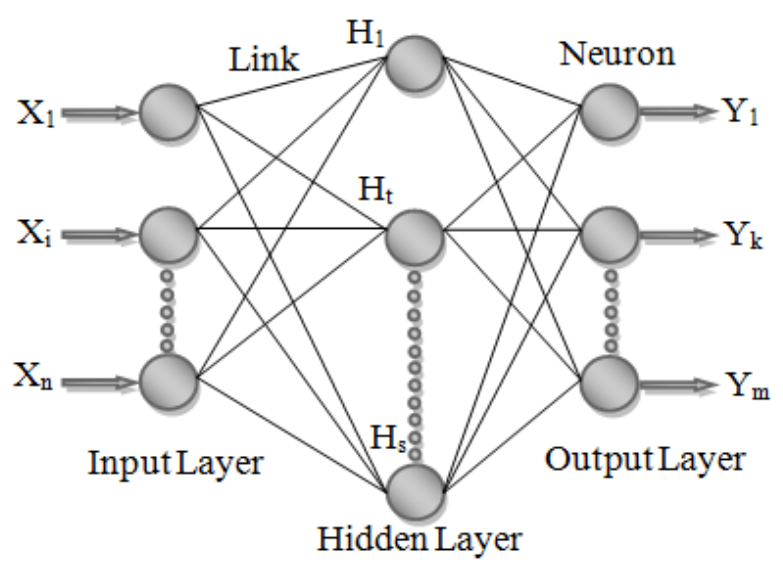

Figure 5. MLP structure

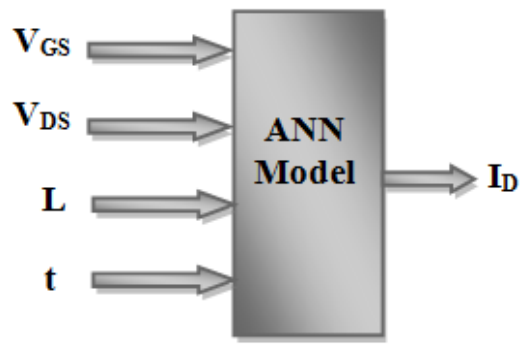

Figure 6. A simplified overview of ANN model

The MLP networks are trained with the standard back-propagation algorithm, which attempts to minimize the error at each iteration and the outputs are compared with the target values to derive the error value. The output of the network is the drain current and the training data consists of set representative data points obtained from the experimental measurements (H. Klauk et al. [9]). The minimum and maximum data ranges used for ANN model are shown in Table 2.

Table 2. Data ranges of the ANN model

\begin{tabular}{|c|c|c|c|c|c|}
\hline \multirow{2}{*}{ Range } & \multicolumn{4}{|c|}{ Input } & Output \\
\hline & Thickness, t (nm) & $L(\mu \mathrm{m})$ & $V_{G S}(V)$ & $V_{D S}(V)$ & $\mathbf{I}_{\mathrm{D}}(\boldsymbol{\mu A})$ \\
\hline Min & 5.7 & 10 & -3.0 & -3.0 & -4.96 \\
\hline Max & 6 & 50 & 0 & 0 & 0 \\
\hline
\end{tabular}

To get a neural network model for the transistor, we examine many different structures with one and three hidden layers and 2 to 15 neurons in each layer. Table 3, shows the result obtained for different structure. The MLP model with 4-9-10-1 Architecture (4 inputs, 9 neurons in first hidden layer, 10 neurons in second hidden layer and one output) resulted in highest accuracy in comparison with the experimental data. 


\section{Table 3. Percentage mean relative errors for testing results of different ANN} structures

\begin{tabular}{|c|c|}
\hline ANN structure & MRE \% (Test) \\
\hline $4-9-10-1$ & 1.4103 \\
$4-4-4-1$ & 3.6466 \\
$4-20-15-1$ & 1.6637 \\
$4-5-4-4-1$ & 8.3205 \\
$4-9-10-4-1$ & 3.7625 \\
\hline
\end{tabular}

A high accuracy of correlation factor (0.9996) between ANN outputs (predicted, $\mathrm{I}_{\text {pred }}$ ) and the corresponding targets (experimental, $\mathrm{I}_{\exp }$ ) is observed as shown in Figure 7.

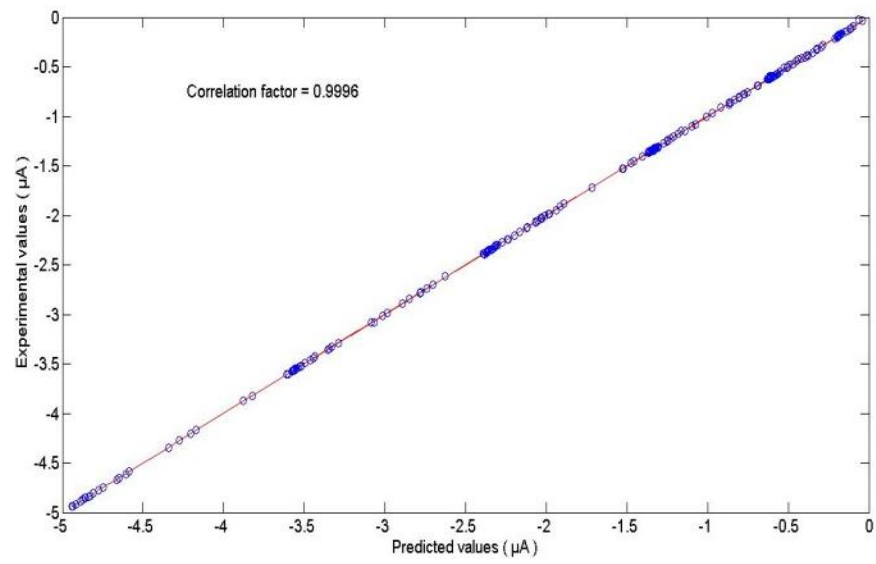

\section{Figure 7. Comparison of the experimental data and predicted (ANN) results for training data}

Figure 8 and 9 show the characteristic family of curves of an OFET for different values of the gate voltage where dashed line represents finite element based Atlas simulation, squares are ANN simulation, and solid line are experimental values manually sampled from [9]. The comparison of current-voltage characteristics $\left(\mathrm{I}_{\mathrm{D}}-\mathrm{V}_{\mathrm{DS}}\right)$ for different $\mathrm{V}_{\mathrm{GS}}$ between experimental data and the proposed ANN model with $\mathrm{L}=10 \mu \mathrm{m}, \mathrm{W}=100 \mu \mathrm{m}$ and $\mathrm{t}=5.7 \mathrm{~nm}$ is shown in Figure 8, the results show that there is a good agreement between the experiment data and predicted values with the least errors. The simulations show a fairly good match especially for the higher gate voltages. The gate and drain voltages have been chosen in such a way that we could be able to make a comparison between published experimental results in [9] and our simulations. 


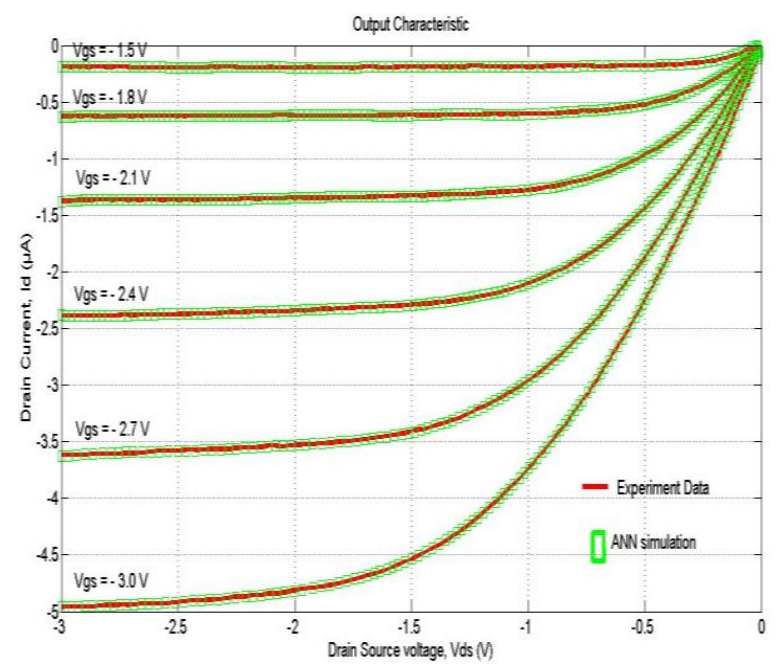

Figure 8. Output characteristic for top contact OFET $(L=10 \mu \mathrm{m}, \mathrm{W}=100 \mu \mathrm{m}$ and $\mathrm{t}$ $=5.7 \mathrm{~nm}$ ) using ANN model

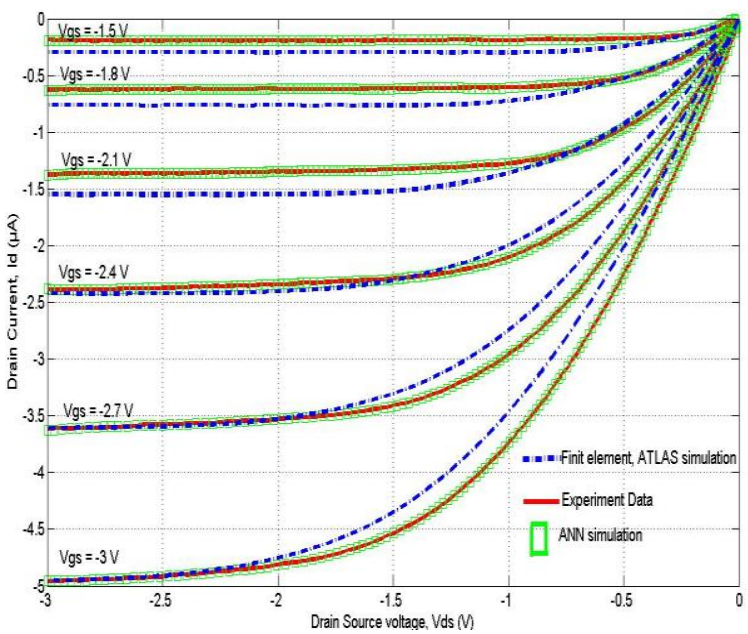

Figure 9. Output characteristic of ANN model and ATLAS simulations

We have simulated the transfer curve for different values of $\mathrm{L}$ ranging from 10 to $50 \mu \mathrm{m}$ (Figure 10), squares are ANN simulation, and solid lines are experimental values manually sampled from [9], the results show that there is a good agreement between the experiment data and ANN predicted values. Figure 11 shows transfer curves of three simulations in the linear region for different values of channel length, the finite element result deviates from the experimental result. This deviation between experiment and simulations might come from the effect of the contact resistance and surface roughness which cannot be included in the current simulation software easily. 


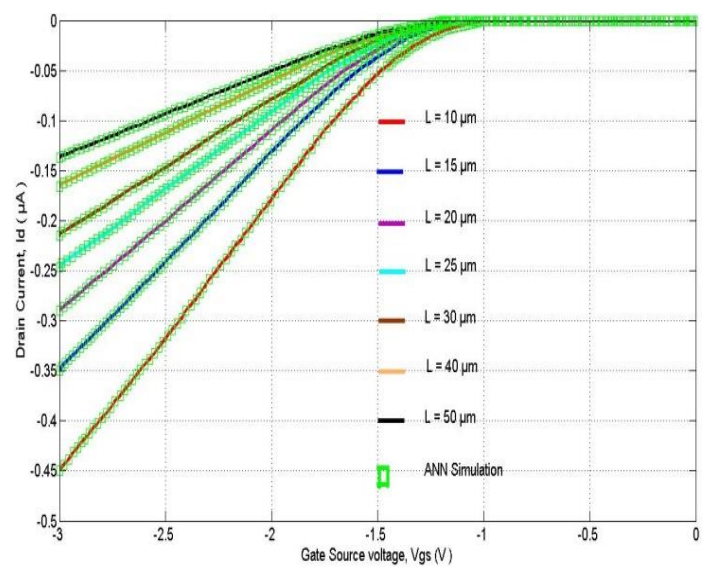

Figure 10. Transfer curves of ANN model for different channel length

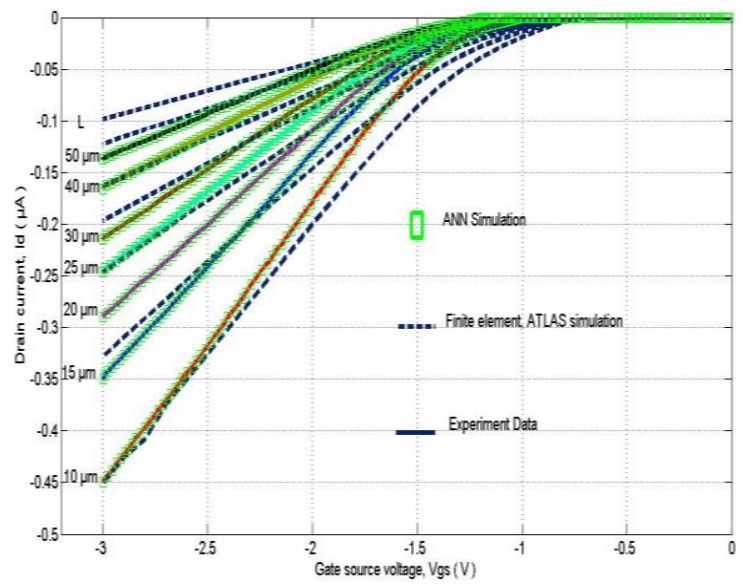

Figure 11. Transfer curves of ANN model and ATLAS simulations for different channel length

\section{Conclusion}

Top contact pentacene OFET has been investigated with both a finite element type simulation and Matlab simulation using artificial neural network (ANN). The comparison between experimental data and predicted (ANN model) values has shown that there is a good agreement between them with the least errors. The physical parameters of the poole-frenkel model used by the finite element are in good agreement with the values presented in published reports. Both simulations agree with the published experimental results.

\section{References}

[1] P. Mach, S. J. Rodriguez, R. Nortrup, P. Wiltzius and J. A. Rogers, "Monolithically integrated, flexible display of polymer dispersed liquid crystal driven by rubber-stamped organic thin-film transistors", Applied Physics Letters, vol. 78, no. 23, (2001), pp. 3592-3594.

[2] T. Someya and T. Sakurai, "Integration of organic field-effect transistors and rubbery pressure sensors for artificial skin applications”, IEEE International Electron Devices Meeting, (2003) December, pp. 203-206.

[3] G. Horowitz, "Organic thin film transistors: From theory to real devices", Journal of Materials Research, vol. 19, issue 7, (2004), pp. 1946-1962.

[4] G. Horowitz, "Organic Field-Effect Transistors", Advanced Materials, vol. 10, (1998), pp. 365-377. 
[5] A. Deman and J. Tardy, "PMMA-Ta2O5 bilayer gate dielectric for low operating voltage organic FETs", science direct Organic Electronics, vol. 6, issue 2, (2005) April, pp. 78-84.

[6] J. B. Koo, J. W. Lim, S. H. Kim, et al., "Pentacene thin-film transistors and inverters with plasma enhanced atomic layer deposited Al2O3 gate dielectric", Thin Solid Films, vol. 515, no. 5, (2007), pp. 3132-3137.

[7] C. Bartic, H. Jansen, A. Campitelli and S. Borghs, "Ta2O5 as gate dielectric material for low-voltage organic thin-film transistors", Organic Electronics, vol. 3, no. 2, (2002), pp. 65-72.

[8] M. Lagziri, R. Picos, N. Bentaibi, M. Boussouis and E. Garcia-Moreno, "Easy Modeling of OTFTs using Neural Networks," Proceedings of the 8th Spanish Conference on Electron Devices, CDE, (2011) February, pp. 1-4

[9] H. Klauk, U. Zschieschang and M. Halik, "Low-voltage organic thin-film transistors with large Transconductance”, J. Appl. Phys, vol. 102, issue 7, (2007), pp. 074514-1 074514-7.

[10] ATLAS User's Manual: Device simulation software, Silvaco International, Santa Clara, CA, (2012) January 18.

[11] S. Mijalkovic, D. Green, A. Nejim, G. Whiting and C. Murphy, "Modeling of Organic Field Effect Transistors for technology and circuit design", 26th International Conference on Microelectronics, (2008) May, pp. 469-476.

[12] C. Shim, F. Maruoka and R. Hattori, "Structural Analysis on Organic Thin-Film Transistor with Device Simulation," IEEE Trans. Electron Devices, vol. 57, no. 1, (2010) January, pp. 195-200.

[13] W. T. Wondmagegn, N. T. Satyala and R. J. Pieper, "Impact of semiconductor/metal interfaces on contact resistance and operating speed of organic thin film transistors", J COMPUT ELECTRON, vol. 10, (2011), pp. 144-153.

[14] A. F. Abo-Elhadeed, "Modeling Ballistic Double Gate MOSFETs using Neural Networks Approach", Proceedings of the 8th Spanish Conference on Electron Devices, CDE, (2011) February, pp. 1-4.

[15] M. Hayati and F.Mozafari, "Modeling and Simulation of Top-Contact Pentacene Thin Film Transistor Using Artificial Neutral Networks", Australian Journal of Basic and Applied Sciences, (2012) September, pp. 242250.

[16] M. Hayati, M. Seifi and A. Rezaei, "Double Gate MOSFET Modeling Based on Adaptive Neuro-Fuzzy Inference System for Nanoscale Circuit Simulation", ETRI Journal, vol. 32, no. 4, (2010) August, pp. 530539. 